\title{
An assessment of plant diversity in Home gardens of Reang Community of Tripura
}

\author{
Dipti Das ${ }^{1}$ and B. K. Datta \\ Plant Taxonomy and Biodiversity Lab., Department of Botany, Tripura University, Suryamaninagar, \\ Tripura West-799022, Tripura, India \\ ${ }^{1}$ Corresponding author, e-mail: diptiagt1964@gmail.com
}

[Received 30.10.2018; Revised 05.12.2018; Accepted 18.12.2018; Published 31.12.2018]

\begin{abstract}
Home gardens are small plots of land surrounding the house and are found in all traditional communities throughout the world. The present study tries to examine the role of Reang Home gardens in North Tripura district of Tripura. A total of 38 Home gardens in 4 hamlet or pada were randomly selected for the study. Total plant inventory and interview method were used to collect data. In Reang Home gardens at North Tripura District overall 148 species under 130 genera belonging to 55 families have been inventoried. Leguminosae and Poaceae were the most dominant families. Forty percent of the Reang Home garden plants were found to harbour edible plant species. (food, Fruits, vegetable, pulses, spices), 22\% medicinal, 18\% ornamental, 4\% timber, $3 \%$ fine wood and other categories remain little over 3\%. Reang Home garden shows a good diversity with multiple uses. Most Reang peoples rely on folk health care traditions. Domestication of wild plants for food, medicine and other purposes are an important activity.
\end{abstract}

Key words: Home garden, Reang tribe, North Tripura

\section{INTRODUCTION}

Home gardens are small plots of land surrounding the house and are found in all traditional communities throughout the world. Home gardens as an ecosystem contains multiple levels of diversity (Watson \& Eyzaguirre 2002). They are the source of edible, medicinal, construction materials and other useful plants, and also act as a repository for uncommon species and varieties. Home gardens play a significant role in maintaining biodiversity and domestication of wild species (Goutam et al. 2006). It is believed that traditional Home Garden practices depend on the type of human community, tradition, needs, beliefs, etc. (Tangjang \& Arunachalam 2009). The traditional Home gardens practiced by ethnic community i.e. Reang community in Tripura, India have been studied to provide a base for further scientific studies.

There is no any optimum or standard size for a Home Garden. The structure of Home Gardens varies in different geographical regions depending upon the socio-economic and ecological conditions. The mean global size for a Home Garden unit varies from about 0.1 to 0.5 hectares (Fernandez \& Nair 1986).

Tripura is a tiny hilly state in North Eastern Region of India. The state is bordered by Bangladesh to the west, south and some part of north, by Assam to extreme north and Mizoram to the east. The North Tripura district is adjacent to Assam and Mizoram. Tripura consists of nineteen diverse ethnic communities of which Tipras are the largest tribe and Reangs are the second largest (Gan Choudhury 2011; Acharyya1999). Reang people are also distributed in the hilly tracts of Chittagong in Bangladesh, Mizoram, Assam and Manipur. 
The correct nomenclature for this ethnic group is Bru means 'man'. (Gan Choudhury 1983). They are mainly distributed in the North Tripura, Dhalai, Gomati and South Tripura district of the state. Generally, Reang peoples live in separate groups or hamlets on hill top. A hamlet is called Kami. But nowadays pada is more commonly used (Gan Choudhury 1983). Kanchanpur of North Tripura district is the highly populated Sub-Division by Reangs in Tripura. They mostly used to practice the Huk or Jhum cultivation. But nowadays they have shifted to modern agricultural practice.

A number of studies on Home gardens have been done by different workers in the world (Soemarwofo 1987; Abdoellah 1990; Perera \& Rajapakse 1991; Michon \& Mary 1994; Agelet et al. 2000; Leiva et al. 2001; Kabir \& Webb 2008; Talemos et al. 2014). Several workers studied Home garden system in India. (Nair \& Sreedharan 1986; Jose \& Shanmugaratnam 1993; Kumar et.al. 1994, Peyre et.al. 2006; Mohan et al.2007; Thomas \& Kurien 2013; Pandey et al. 2007; Kala 2010; Bajpai et al. 2013). In Northeast India, several workers have worked on traditional Home gardening system (Borthakur et al. 1998; Samati 2004; Das \& Das 2005; Devi \& Das 2010, 2013; Tangjang \& Arunachalam 2009; Sahoo et al. 2010). However, there is no available literature on Home Gardening system of Tripura. Therefore, current study was undertaken to provide a baseline information on the Home Garden system of Reang tribe of Tripura. The main objective of the present study is to make a detailed inventory of floristic composition and document the utilization pattern of Home garden plants of Reang tribe of Tripura.

\section{MATERIALS AND METHODS}

\section{Study Area:}

The study was conducted in four villages namely, Dakshin Jariham para $\left(24^{\circ} 2^{\prime} 13.4^{\prime \prime} \mathrm{N}\right.$, $92^{\circ} 12^{\prime} 9.5^{\prime \prime}$ E, Elevation $\left.111 \mathrm{~m}\right)$, Bru para $\left(23^{\circ} 24^{\prime} 59.4^{\prime \prime} \mathrm{N}, 91^{\circ} 47^{\prime} 51.4^{\prime \prime}\right.$ E, Elevation $\left.155 \mathrm{~m}\right)$ Bhaktamohan para $\left(24^{\circ} 04^{\prime} 19.4^{\prime \prime} \mathrm{N}, 9^{\circ} 13^{\prime} 13.7^{\prime \prime}\right.$ E, Elevation $\left.132 \mathrm{~m}\right)$ and Saboal (235 $56^{\prime} 45.2^{\prime \prime}$ N, 92 $12^{\prime} 29^{\prime \prime}$,E,Elevation714 m) of Kanchanpur Sub-Division of North Tripura district during October 2014 to September 2016 in different seasons. A total of 38 households were surveyed.

In the survey sites there are three types of land uses:

i. Forest land: Land deeds distributed according to Forest Right act 2006 (Patta)

ii. Joteoe land; and

iii. Khas land.

Dakshin Jariham para falls in the first category of land use. Bru para and Bhaktamohan para falls in the second category. In Saboal, people reside in jote land under the name of orange grower society and some are residing in forest land (Patta system). In the third category, people uses Khas land for Jhum and shifting cultivation and resides temporarily in Choto Kangrai, Bara Kangrai and Hamlaia para, however, shifting cultivation is not included in the data collected. In Bru para, Jariham para and Saboal, Home gardens were established in open areas and in Bhaktamohan para few Home Gardens were with fence.

A detailed survey of household was conducted and relevant information about the Home Gardens were collected through personal interview method and prepared questionnaires. Collected specimens were processed into mounted herbarium-sheets following standard methods (Jain \& Rao 1977) and was deposited in Tripura University Herbarium. Plants were identified using Flora of Tripura State(Deb 1981, 1983), Flora of Assam ( Kanjilal et al. 1934 - 1940), Bengal Plants (Prain 1903), The Flora of British India (Hooker 
$1872-1897)$ and existing different literatures. Few village markets were also surveyed to record the Home Garden products. The nomenclatures were updated using the Plant list (www.theplantlist.org) for the recorded plant species. The plant species have been arranged alphabetically and their details presented in Table 1.

\section{RESULTS AND DISCUSSION}

To assess the tribal Home Gardens oral informations were collected through self structured questionnaire containing - size of Home gardens, reasons for gardening, age of the garden, any investment, income generated from gardening, management practise etc,.The informantion regarding medicinal uses, parts used and mode of preparation and administration were also recorded.

The Home Gardens of Reang community in North Tripura District altogether constitute 148 species under 130 genera belonging to 55 families (Table 1; Figure 1). Among the 55 families Leguminosae and Poaceae were the most dominant with 13 species each. Apocynaceae, Euphorbiaceae and Lamiaceae remained second dominant families with 8 plant species each. Cucurbitaceae and Solanaceae were the third dominant families with 6 species each (Figure 2). In this study plant species recorded were categorised under different life forms- climbers, herbs, shrubs, small trees, trees, and woody climbers. Different economic categories were found mainly food (vegetables, fruits, grains, spices, etc.), medicinal, ornamental and construction material. The main useful parts were fruits and leaves (Figure 4).

Table 1. Enumeration of plant species inventoried in Home gardens, including their common name and local use

\begin{tabular}{|c|c|c|c|c|}
\hline Scientific Name [Family]; specimen cited & Reang Name & Habit & Parts Used & Uses \\
\hline $\begin{array}{l}\text { Acacia auriculiformis Benth.; [Leguminosae]; } \\
\text { TUH } 1827 .\end{array}$ & & Tree & Wood & Fuel wood \\
\hline $\begin{array}{l}\text { Achyranthes aspera L. [Amaranthaceae]; TUH } \\
1778 .\end{array}$ & & Herb & Whole plant & Medicinal \\
\hline $\begin{array}{l}\text { Acmella paniculata (Wall. ex DC.) R.K. Jansen } \\
\text { [Asteraceae]; TUH } 1701 .\end{array}$ & Ushnoi & Herb & Inflorescence & Medicinal \\
\hline $\begin{array}{l}\text { Aegle marmelos (L.) Corrêa [Rutaceae]; TUH } \\
1791 .\end{array}$ & $\mathrm{Bel}$ & Tree & Fruits, leaves & Fruit, Ritual \\
\hline Agave americana L. [Asparagaceae]; TUH 1828. & & Herb & Whole plant & Ornamental \\
\hline $\begin{array}{l}\text { Albizia lebbeck (L.) Benth. [Leguminosae]; TUH } \\
1792\end{array}$ & Bolphu & Tree & Timber & Timber \\
\hline $\begin{array}{l}\text { Albizia procera(Roxb.)Benth. [Leguminosae]; } \\
\text { TUH } 1833\end{array}$ & Khouribufang & Tree & Timber & Timber \\
\hline $\begin{array}{l}\text { Allamanda cathartica L. [Apocynaceae]; TUH } \\
1793 .\end{array}$ & & Shrub & Flower & Ornamental \\
\hline $\begin{array}{l}\text { Alocasia macrorrhizos (L.) G.Don [Araceae]; } \\
\text { TUH } 1829\end{array}$ & Thakortoma & Herb & Rootstock & Vegetable \\
\hline $\begin{array}{l}\text { Aloe vera (L.) Burm.f. [Xanthorrhoeaceae]; TUH } \\
1836\end{array}$ & & Herb & Leaves & Medicinal \\
\hline $\begin{array}{l}\text { Alpinia nigra (Gaertn.) Burtt [Zingiberaceae]; } \\
\text { TUH } 1857\end{array}$ & Tara & Herb & Stem & $\begin{array}{l}\text { Vegetable, } \\
\text { Medicinal }\end{array}$ \\
\hline $\begin{array}{l}\text { Alstonia scholaris (L.) R.Br. [Apocynaceae]; } \\
\text { TUH } 1850\end{array}$ & Chethuang & Tree & Bark & Medicinal \\
\hline $\begin{array}{l}\text { Alternanthera brasiliana (L.) Kuntze } \\
\text { [Amaranthaceae];TUH } 1861\end{array}$ & Lal agunsita & Herb & Leaves & Medicinal \\
\hline $\begin{array}{l}\text { Amorphophallus bulbifer (Roxb.) Blume } \\
\text { [Araceae]; TUH } 1854\end{array}$ & Batema & Herb & $\begin{array}{l}\text { Corm and } \\
\text { daughter corm }\end{array}$ & Vegetable \\
\hline $\begin{array}{l}\text { Ananas comosus (L.) Merr. [Bromeliaceae]; } \\
\text { TUH } 1864\end{array}$ & Omtoi & Herb & Fruits,leaves & Fruit, Medicinal \\
\hline
\end{tabular}


Dipti Das \& B.K. Datta 211

\begin{tabular}{|c|c|c|c|c|}
\hline Scientific Name [Family]; specimen cited & Reang Name & Habit & Parts Used & Uses \\
\hline $\begin{array}{l}\text { Andrographis paniculata (Burm.f.) Nees } \\
\text { [Acanthaceae]; TUH } 1865\end{array}$ & & Herb & Leaves & Medicinal \\
\hline Annona squamosa L. [Annonaceae]; TUH 1898 & & Tree & Fruits, leaves & Fruit, Medicinal \\
\hline Areca catechu L. [Arecaceae]; TUH 1851 & Kuwai & Small tree & Fruits & Masticator \\
\hline $\begin{array}{l}\text { Artocarpus chamaBuch.-Ham. [Moraceae]; TUH } \\
1856\end{array}$ & & Tree & Wood, Fruit & Timber, Fruit \\
\hline $\begin{array}{l}\text { Artocarpus heterophyllus Lam. [Moraceae]; } \\
\text { TUH } 1740 .\end{array}$ & Thaiphung & Tree & Fruit,Wood & Fruit, Timber \\
\hline $\begin{array}{l}\text { Artocarpus lacucha Buch.-Ham. [Moraceae]; } \\
\text { TUH } 1810\end{array}$ & Dua & Tree & Wood, fruit & Timber, Fruit \\
\hline $\begin{array}{l}\text { Asparagus racemosus Willd. [Asparagaceae]; } \\
\text { TUH } 1855\end{array}$ & Suptisara & Climber & Root & Medicinal \\
\hline $\begin{array}{l}\text { Ayapana triplinervis (Vahl) R.M.King \& H.Rob. } \\
\text { [Asteraceae]; TUH } 1720 .\end{array}$ & Maisnoi & Herb & Leaves & Medicinal \\
\hline Bambusa balcooaRoxb.[Poaceae];TUH 1858 & Wamlang & Herb & Shoot, culm & $\begin{array}{l}\text { Vegetable, } \\
\text { Construction } \\
\text { material }\end{array}$ \\
\hline $\begin{array}{l}\text { Bambusa cacharensisR.B.Majumdar [Poaceae]; } \\
\text { TUH } 1866\end{array}$ & & Herb & Culm & Miscellaneous \\
\hline $\begin{array}{l}\text { Bambusa polymorpha Munro [Poaceae]; TUH } \\
1859\end{array}$ & Woashur & Herb & Culm & $\begin{array}{l}\text { Construction } \\
\text { material }\end{array}$ \\
\hline Bambusa tuldaRoxb. [Poaceae]; TUH 1897 & Woarna & Herb & Shoot, culm & $\begin{array}{l}\text { Vegetable, } \\
\text { Cottage industry }\end{array}$ \\
\hline Basella alba L. [Basellaceae]; TUH 1830 & Muifrai & Herb & $\begin{array}{l}\text { Leaves, stem, } \\
\text { inflorescence }\end{array}$ & Vegetable \\
\hline Bauhinia variegata L. [Leguminosae];TUH 1832 & & Tree & Flower & Ornamental \\
\hline $\begin{array}{l}\text { Benincasa hispida } \text { (Thunb.) Cogn. } \\
\text { [Cucurbitaceae]; TUH } 1896\end{array}$ & Khaklu & Herb & $\begin{array}{l}\text { Fruit, leaves, } \\
\text { stem }\end{array}$ & Vegetable \\
\hline Bombax ceiba L. [Malvaceae]; TUH1885 & $\begin{array}{l}\text { Bachum } \\
\text { bufang }\end{array}$ & Tree & Fibre of fruit & $\begin{array}{l}\text { Stuffing } \\
\text { material }\end{array}$ \\
\hline $\begin{array}{l}\text { Bougainvillea glabra Choicy [Nyctaginaceae]; } \\
\text { TUH1895 }\end{array}$ & Khunglekha & $\begin{array}{l}\text { Woody } \\
\text { climber }\end{array}$ & Flower & Ornamental \\
\hline $\begin{array}{l}\text { Bryophyllum pinnatum (Lam.) Oken } \\
\text { [Crassulaceae]; TUH } 1886\end{array}$ & Jihar & Herb & Leaves & Medicinal \\
\hline $\begin{array}{l}\text { Cajanus cajan (L.) Millsp. [Leguminosae]; TUH } \\
1723 .\end{array}$ & Khakleing & Shrub & Fruit & Vegetable \\
\hline $\begin{array}{l}\text { Caladium bicolor (Aiton) Vent. [Araceae]; TUH } \\
1899\end{array}$ & & Herb & Leaves & Ornamental \\
\hline Calamus tenuis Roxb. [Arecaceae]; TUH 1860 & Raikecha & Climber & Stem & Miscellaneous \\
\hline $\begin{array}{l}\text { Calamus viminalis Willd. [Arecaceae]; TUH } \\
1868\end{array}$ & Chalang & Climber & Stem & $\begin{array}{l}\text { Making } \\
\text { handicrafts }\end{array}$ \\
\hline $\begin{array}{l}\text { Callicarpa arborea Roxb. [Lamiaceae]; TUH } \\
1886\end{array}$ & Chamathai & Small tree & Stem & Fire wood \\
\hline Canna indica $\mathrm{L}$. [Cannaceae]; TUH1894 & & Herb & Flower & Ornamental \\
\hline Capsicum annuum L. [Solanaceae]. TUH 1702. & Thamosa & Herb & Fruit & Spices \\
\hline Carica papaya L. [Caricaceae]; TUH 1862 & Kingkoya & Herb & Fruit & Fruit, vegetable \\
\hline $\begin{array}{l}\text { Cascabela thevetia (L.) Lippold [Apocynaceae]; } \\
\text { TUH } 1893\end{array}$ & Khmbarungjak & Shrub & Flower & Ornamental \\
\hline $\begin{array}{l}\text { Catharanthus roseus (L.) G.Don [Apocynaceae]; } \\
\text { TUH } 1887\end{array}$ & Khumbairagi & Herb & Flower & Ornamental \\
\hline $\begin{array}{l}\text { Celosia argentea } \mathrm{L} . \text { [Amaranthaceae]; TUH } \\
1716 .\end{array}$ & Khumchak & Herb & Inflorescence & Ornamental \\
\hline $\begin{array}{l}\text { Centratherum punctatum Cass. [ Asteraceae]; } \\
\text { TUH } 1831\end{array}$ & & Herb & Flower & Ornamental \\
\hline
\end{tabular}


212 Plant diversity in Reang Home Gardens in Tripura

\begin{tabular}{|c|c|c|c|c|}
\hline Scientific Name [Family]; specimen cited & Reang Name & Habit & Parts Used & Uses \\
\hline $\begin{array}{l}\text { Cheilocostus speciosus (J.Koenig) C.D. Specht } \\
\text { [Costaceae]; TUH } 1852\end{array}$ & & Herb & Rhizome & Medicinal \\
\hline Citrus limon (L.) Osbeck [Rutaceae]; TUH 1834 & Slung & Small tree & Fruit & Fruit \\
\hline $\begin{array}{l}\text { Citrus maxima (Burm.) Merr. [Rutaceae]; TUH } \\
1869\end{array}$ & Jamra & Small tree & Fruit & Fruit \\
\hline Citrus reticulata Blanco [Rutaceae]; TUH1888 & Slung & Small tree & Fruit & Fruit \\
\hline Citrus hystrix DC. [Rutaceae] TUH1891 & Satkara & Small tree & Fruit & $\begin{array}{l}\text { Fruit juice used } \\
\text { in curry, } \\
\text { medicinal }\end{array}$ \\
\hline $\begin{array}{l}\text { Clerodendrum infortunatum } \mathrm{L} \text {. [Lamiaceae]; } \\
\text { TUH } 1801\end{array}$ & Chukhuima & Shrub & Root & Medicinal \\
\hline Cocos nucifera L. [Arecaceae]; TUH1889 & Naningra & Small tree & Fruit, leaves & $\begin{array}{l}\text { Fruit, broom } \\
\text { making }\end{array}$ \\
\hline $\begin{array}{l}\text { Codiaeum variegatum (L.) Rumph. ex A.Juss. } \\
\text { [Euphorbiaceae]; TUH } 1870\end{array}$ & & Shrub & Leaves & Ornamental \\
\hline $\begin{array}{l}\text { Combretum indicum }(\mathrm{L} .) \text { De Filipps } \\
\text { [Combretaceae]; TUH } 2401\end{array}$ & & $\begin{array}{l}\text { Woody } \\
\text { climber }\end{array}$ & Flower & Ornamental \\
\hline $\begin{array}{l}\text { Cosmos sulphureus Cav. [Asteraceae]; TUH } \\
2418\end{array}$ & Khumtai & Herb & Flower & Ornamental \\
\hline $\begin{array}{l}\text { Crinum asiaticum L. [Amaryllidaceae]; TUH } \\
2467\end{array}$ & & Herb & Flower & Ornamental \\
\hline $\begin{array}{l}\text { Cuphea viscosissima Jacq. [Lythraceae]; TUH } \\
1706 .\end{array}$ & & Herb & Whole plant & Weed \\
\hline Curcuma longa L. [Zingiberaceae]; TUH 1835 & Korma & Herb & Rhizome & $\begin{array}{l}\text { Spices, } \\
\text { Medicinal }\end{array}$ \\
\hline $\begin{array}{l}\text { Cymbopogon citratus (DC.) Stapf [Poaceae]; } \\
\text { TUH } 1871\end{array}$ & Suimander & Herb & Leaves & Spices \\
\hline Datura metel L. [Solanaceae]; TUH 2402 & Dhutra & Shrub & Leaves & Medicinal \\
\hline $\begin{array}{l}\text { Delonix regia (Hook.) Raf. [Leguminosae]; TUH } \\
2417\end{array}$ & & Tree & Wood & Fire wood \\
\hline $\begin{array}{l}\text { Dendrocalamus longispathus (Kurz) Kurz } \\
\text { [Poaceae]; TUH } 2435\end{array}$ & Wamli & Herb & Shoot, & Vegetable \\
\hline Dioscorea alata L. [Dioscoreaceae]; TUH 2466 & Thamtai & Climber & Root tuber & Vegetable \\
\hline $\begin{array}{l}\text { Dioscorea bulbifera L. [Dioscoreaceae]; TUH } \\
1710 .\end{array}$ & & Climber & Bulbils & Vegetable \\
\hline Dolichos lablab L. [Leguminosae]; TUH 2441 & Kasoi & Climber & Fruits & Vegetable \\
\hline Eryngium foetidum L. [Apiaceae]; TUH 2455 & & Herb & Leaves & Spices \\
\hline $\begin{array}{l}\text { Erythrina variegata L. [Leguminosae]; TUH } \\
2403\end{array}$ & Mander & Tree & Wood & Fuel wood \\
\hline Euphorbia hirta L. [Euphorbiaceae]; TUH 2446 & Howsaifa bithi & Herb & $\begin{array}{l}\text { young whole } \\
\text { plant }\end{array}$ & Medicinal \\
\hline $\begin{array}{l}\text { Euphorbia nerifolia } \text { L. [Euphorbiaceae]; TUH } \\
2415\end{array}$ & Shiblatha & Small tree & latex, leaves & Medicinal \\
\hline $\begin{array}{l}\text { Euphorbia tithymaloides L. [Euphorbiaceae]; } \\
\text { TUH } 2433\end{array}$ & & Herb & Whole plant & Ornamental \\
\hline Ficus hispida L.f. [Moraceae]; TUH 2465 & Thaibeiche & Small tree & $\begin{array}{l}\text { Leaves, } \\
\text { inflorescence }\end{array}$ & $\begin{array}{l}\text { Fodder, } \\
\text { Vegetable }\end{array}$ \\
\hline $\begin{array}{l}\text { Gardenia jasminoides J.Ellis [Rubiaceae]; TUH } \\
2425\end{array}$ & & Shrub & Flower & Ornamental \\
\hline Garuga pinnata Roxb. [Burseraceae]; TUH 2447 & Nilfung & Tree & Leaves & Medicinal \\
\hline Gloriosa superba L. Colchicaceae; TUH 2404 & & Herb & Flower & Ornamental \\
\hline Gmelina arborea Roxb. [Lamiaceae]; TUH 2423 & Kambarui & Tree & Wood & Timber \\
\hline Gossypium hirsutam L. [Malvaceae]; TUH2464 & Khul & Shrub & Lints from seeds & Multipurpose \\
\hline $\begin{array}{l}\text { Hemidesmus indicus (L.) R. Br. ex Schult. } \\
\text { [Apocynaceae]; TUH2445 }\end{array}$ & & Herb & root & Medicinal \\
\hline $\begin{array}{l}\text { Hibiscus rosa-sinensis L. [Malvaceae]; TUH } \\
2414\end{array}$ & & Shrub & Flower & Ornamental \\
\hline $\begin{array}{l}\text { Holmskioldia sanguinea Retz. [Lamiaceae], } \\
\text { TUH } 1703 .\end{array}$ & & Shrub & Flower & Ornamental \\
\hline
\end{tabular}


Dipti Das \& B.K. Datta 213

\begin{tabular}{|c|c|c|c|c|}
\hline Scientific Name [Family]; specimen cited & Reang Name & Habit & Parts Used & Uses \\
\hline $\begin{array}{l}\text { Homalomena aromatica (Spreng .) Schott } \\
\text { [Araceae]; TUH } 2454\end{array}$ & Kamaitru & Herb & petiole & Vegetable \\
\hline Hyptis capitata Jacq. [Lamiaceae]; TUH 1727. & $\begin{array}{l}\text { Maishnoiktor } \\
\text { ma }\end{array}$ & Herb & Leaves & Medicinal \\
\hline $\begin{array}{l}\text { Impatiens balsamina L. [Balsaminaceae]; TUH } \\
2405\end{array}$ & & Herb & Flower & Ornamental \\
\hline $\begin{array}{l}\text { Imperata cylindrica (L.) Raeusch [Poaceae]; } \\
\text { TUH } 2444 .\end{array}$ & & Herb & Leaves & House making \\
\hline $\begin{array}{l}\text { Ipomoea cairica (L.) Sweet [Convolvulaceae]; } \\
\text { TUH } 1796 .\end{array}$ & & Herb & Flower & Ornamental \\
\hline $\begin{array}{l}\text { Jasminum sambac (L.) Aiton [Oleaceae]; TUH } \\
1786 .\end{array}$ & & Shrub & Flower & Ornamental \\
\hline Jatropha curcas L. [Euphorbiaceae]; TUH 2406 & $\begin{array}{l}\text { Chukhuima } \\
\text { fang }\end{array}$ & Herb & Latex & Medicinal \\
\hline $\begin{array}{l}\text { Jatropha gossypiifolia L. [Euphorbiaceae]; TUH } \\
2434\end{array}$ & & Shrub & Latex & Medicinal \\
\hline $\begin{array}{l}\text { Justicia gendarussa Burm.f. [Acanthaceae]; } \\
\text { TUH2452 }\end{array}$ & & $\begin{array}{l}\text { Under } \\
\text { shrub }\end{array}$ & Leaves & Medicinal \\
\hline $\begin{array}{l}\text { Lagenaria siceraria (Molina) Standl. } \\
\text { [Cucurbitaceae]; TUH } 2413\end{array}$ & Muilao & Herb & Fruits, twigs & Vegetables \\
\hline Litchi chinensis Sonn. [Sapindaceae]; TUH 2443 & Lechu & Tree & Fruits & Fruit \\
\hline $\begin{array}{l}\text { Luffa acutangula (L.) Roxb. [Cucurbitaceae]; } \\
\text { TUH } 1787\end{array}$ & Chenga & Herb & Fruits & Vegetable \\
\hline $\begin{array}{l}\text { Luffa cylindrica (L.) M.Roem. [Cucurbitaceae]; } \\
\text { TUH } 1719 .\end{array}$ & Fra-mathai & Herb & Fruits & Vegetable \\
\hline $\begin{array}{l}\text { Magnolia champaca (L.) Baill. ex Pierre } \\
\text { [Magnoliaceae]; TUH } 2407\end{array}$ & & Tree & Flower & Ornamental \\
\hline $\begin{array}{l}\text { Mangifera indica L. [Anacardiaceae]; TUH } \\
1738 .\end{array}$ & Thaichuk & Tree & Fruits & Fruit \\
\hline $\begin{array}{l}\text { Manihot esculenta Crantz [Euphorbiaceae];TUH } \\
1847\end{array}$ & Thabachu & Shrub & Root tuber & Vegetable \\
\hline $\begin{array}{l}\text { Maranta arundinacea L. [Marantaceae]; TUH } \\
2442\end{array}$ & & Herb & rhizome & Medicinal \\
\hline Melia azedarach L. [Meliaceae]; TUH 2459 & Eaing & Tree & Leaves & Medicinal \\
\hline $\begin{array}{l}\text { Melocanna baccifera (Roxb.)Kurz. [Poaceae]; } \\
\text { TUH } 2408\end{array}$ & Warthoi & Herb & Shoot, culm & $\begin{array}{l}\text { Vegetable, Hut } \\
\text { making }\end{array}$ \\
\hline Mesua ferrea L. [Calophyllaceae]; TUH 2463 & & Tree & Whole plant & Ornamental \\
\hline $\begin{array}{l}\text { Momordica dioica Roxb. ex Willd. } \\
\text { [Cucurbitaceae]; TUH } 1848\end{array}$ & & Herb & Fruits & Vegetable \\
\hline $\begin{array}{l}\text { Morinda angustifolia Roxb. [Rubiaceae]; TUH } \\
2412\end{array}$ & & Small tree & Root & Medicinal \\
\hline $\begin{array}{l}\text { Moringa oleifera Lam. [Moringaceae]; TUH } \\
2457\end{array}$ & Sejana & Small tree & $\begin{array}{l}\text { Fruit, leaves, } \\
\text { Flowers }\end{array}$ & Vegetable \\
\hline Morus australis Poir. [Moraceae]; TUH 1789. & & Small tree & Fruits & Medicinal \\
\hline Musa paradisiaca L. [Musaceae]; TUH 2460 & Thalik & Herb & $\begin{array}{l}\text { Shoot, Fruits, } \\
\text { Inflorescence }\end{array}$ & Fruit, vegetable \\
\hline $\begin{array}{l}\text { Nyctanthes arbor-tristis L. [Oleaceae]; TUH } \\
2409\end{array}$ & Shephali & Small tree & Flowers, leaves & $\begin{array}{l}\text { Ornamental, } \\
\text { medicinal }\end{array}$ \\
\hline $\begin{array}{l}\text { Ocimum tenuiflorum L. [Lamiaceae]; TUH } \\
1788 .\end{array}$ & & Herb & Leaves & Medicinal \\
\hline $\begin{array}{l}\text { Oldenlandia verticillata } \text { L. [Rubiaceae]; TUH } \\
2461\end{array}$ & & Herb & Leaves & Medicinal \\
\hline $\begin{array}{l}\text { Oroxylum indicum Vent. [Bignoniaceae]; TUH } \\
2410\end{array}$ & Takharung & Tree & Fruits, Bark & $\begin{array}{l}\text { Vegetable, } \\
\text { medicinal. }\end{array}$ \\
\hline $\begin{array}{l}\text { Pachyrhizus erosus (L.) Urb. [Leguminosae]; } \\
\text { TUH } 1741 .\end{array}$ & Khakchangma & Climber & Root tuber & Raw food \\
\hline $\begin{array}{l}\text { Pandanus amaryllifolius Roxb. [Pandanaceae]; } \\
\text { TUH } 1846\end{array}$ & & Herb & Leaves & $\begin{array}{l}\text { Spices, } \\
\text { medicinal }\end{array}$ \\
\hline $\begin{array}{l}\text { Parkia javanica(Lam.) Merr. [Leguminosae]; } \\
\text { TUH } 2448\end{array}$ & Wikre & Tree & $\begin{array}{l}\text { Fruits, } \\
\text { Inflorescence }\end{array}$ & Vegetable \\
\hline
\end{tabular}


214 Plant diversity in Reang Home Gardens in Tripura

\begin{tabular}{|c|c|c|c|c|}
\hline Scientific Name [Family]; specimen cited & Reang Name & Habit & Parts Used & Uses \\
\hline Passiflora foetida L. [Passifloraceae]; TUH 2411. & duthaithoi & Herb & Ripe fruits & Fruit \\
\hline Phaseolus lunatus L. [Leguminosae]; TUH 1717. & & Climber & Seeds & Pulses \\
\hline $\begin{array}{l}\text { Phragmites karka (Retz.) Trin. ex Steud. } \\
\text { [Poaceae]; TUH } 1842\end{array}$ & Lubong & Herb & Inflorescence & Rituals \\
\hline $\begin{array}{l}\text { Phyllanthus acidus (L.) Skeels [Euphorbiaceae]; } \\
\text { TUH } 2429\end{array}$ & & Tree & Fruit & Fruit \\
\hline $\begin{array}{l}\text { Phyllanthus emblica L. [Phyllanthaceae]; TUH } \\
2458\end{array}$ & Amlai & Tree & Fruits & Raw fruit \\
\hline Physalis minima L. [Solanaceae]; TUH 1790. & Thaithoi & Herb & Ripe fruit & Fruit \\
\hline Piper betle L. [Piperaceae]; TUH 2462 & Fathoi & Climber & Leaves & Masticator \\
\hline $\begin{array}{l}\text { Plectranthus scutellarioides (L.) R.Br. } \\
\text { [Lamiaceae]; TUH } 2411\end{array}$ & & Herb & whole plant & Ornamental \\
\hline $\begin{array}{l}\text { Plumbago zeylanica L. [Plumbaginaceae]; TUH } \\
1817 .\end{array}$ & Agunsita & Herb & Leaves & Medicinal \\
\hline Plumeria rubra L. [Apocynaceae]; TUH 1880 & & Tree & Flowers & Ornamental \\
\hline Psidium guajava L. [Myrtaceae]; TUH 1900 & Guiang & Small tree & Fruits & Fruits \\
\hline $\begin{array}{l}\text { Rauvolfia serpentina (L.) Benth. ex Kurz } \\
\text { [Apocynaceae]; TUH } 1747 .\end{array}$ & Chanduama & Herb & Roots & Medicinal \\
\hline Rosa centifolia L. [Rosaceae]; TUH 1884 & & Shrub & Flowers & Ornamental \\
\hline $\begin{array}{l}\text { Sansevieria trifasciata Prain [Asparagaceae]; } \\
\text { TUH } 1890\end{array}$ & Musaslai & Herb & Rhizome & Medicinal \\
\hline $\begin{array}{l}\text { Schizostachyum dullooa (Gamble) } \\
\text { R.B.Majumdar [Poaceae]; TUH } 1843\end{array}$ & Wakla & Herb & Culm & $\begin{array}{l}\text { Handicrafts, } \\
\text { small } \\
\text { construction }\end{array}$ \\
\hline $\begin{array}{l}\text { Sechium edule (Jacq.) Sw [Cucurbitaceae]; TUH } \\
1879 .\end{array}$ & & Climber & Fruit & Vegetable \\
\hline $\begin{array}{l}\text { Senna siamea (Lam.) H.S.Irwin \& Barneby } \\
\text { [Leguminosae]; TUH } 1883\end{array}$ & Muitapi & Tree & Whole plant & Fire wood \\
\hline $\begin{array}{l}\text { Setaria italica (L.) P.Beauv. [Poaceae]; TUH } \\
1844\end{array}$ & Maisoii & Herb & Grains & Food \\
\hline Sida rhombifolia L. [Malvaceae]; TUH 1875 & Dangarnusi & Herb & Whole plant & Broom \\
\hline Solanum melongena $\mathrm{L}$. [Solanaceae]; TUH 1882 & Fanthau & Undershrub & Fruits & Vegetable \\
\hline Solanum torvum Sw. [Solanaceae]; TUH 1840 & Khanka & Shrub & Fruits & Vegetable \\
\hline $\begin{array}{l}\text { Solanum violaceum Ortega [Solanaceae]; TUH } \\
1730 .\end{array}$ & Khanka & Undershrub & Fruits & Vegetable \\
\hline $\begin{array}{l}\text { Stephania glabra (Roxb.) Miers } \\
\text { [Menispermaceae]; TUH } 1762 \text {. }\end{array}$ & $\begin{array}{l}\text { Blong Kha- } \\
\text { kchangma,Tha } \\
\text { nda manik }\end{array}$ & Herb & Leaves & Medicinal \\
\hline Sterculia villosa Roxb. [Malvaceae]; TUH 1838 & Fathi & Tree & Bark & Rope making \\
\hline $\begin{array}{l}\text { Syzygium cumini (L.) Skeels [Myrtaceae]; TUH } \\
1878\end{array}$ & Chambu & Tree & Fruits & Fruit \\
\hline $\begin{array}{l}\text { Tabernaemontana divaricata (L.) R.Br. ex } \\
\text { Roem. \& Schult. [Apocynaceae]; TUH } 1841\end{array}$ & & Shrub & Flowers & Ornamental \\
\hline Tagetes erecta L. [Asteraceae]; TUH 1873 & Khumtu & Herb & Flowers & Ornamental \\
\hline $\begin{array}{l}\text { Tamarindus indica L. [Leguminosae]; TUH } \\
1758 .\end{array}$ & Tintoi & Tree & Fruits & Multipurpose \\
\hline Tectona grandis L.f. [Lamiaceae]; TUH 1874 & Segun & Tree & Woody stem & Timber \\
\hline $\begin{array}{l}\text { Terminalia bellirica (Gaertn.) Roxb. } \\
\text { [Combretaceae]; TUH } 1839\end{array}$ & Thaisatoi & Tree & Fruits & Medicinal \\
\hline $\begin{array}{l}\text { Thunbergia grandiflora (Roxb. ex Rottl.) Roxb. } \\
\text { [Acanthaceae]; TUH } 1877\end{array}$ & & Climber & Flowers & Ornamental \\
\hline $\begin{array}{l}\text { Thyrsostachys oliveri Gamble [Poaceae]; TUH } \\
1881\end{array}$ & & Herb & Culm & Multipurpose \\
\hline $\begin{array}{l}\text { Thysanolaena latifolia (Roxb. ex Hornem.) } \\
\text { Honda [Poaceae]; TUH } 1872\end{array}$ & Nouksi & Herb & Inflorescence & Broom \\
\hline Toona ciliata M. Roem. [Meliaceae]; TUH 1845 & Puma & Tree & Woody stem & Timber \\
\hline $\begin{array}{l}\text { Xanthosoma sagittifolium (L.) Schott } \\
\text { [Araceae]; TUH } 1863\end{array}$ & Kala manai & Herb & petiole, stolon & Vegetable \\
\hline
\end{tabular}




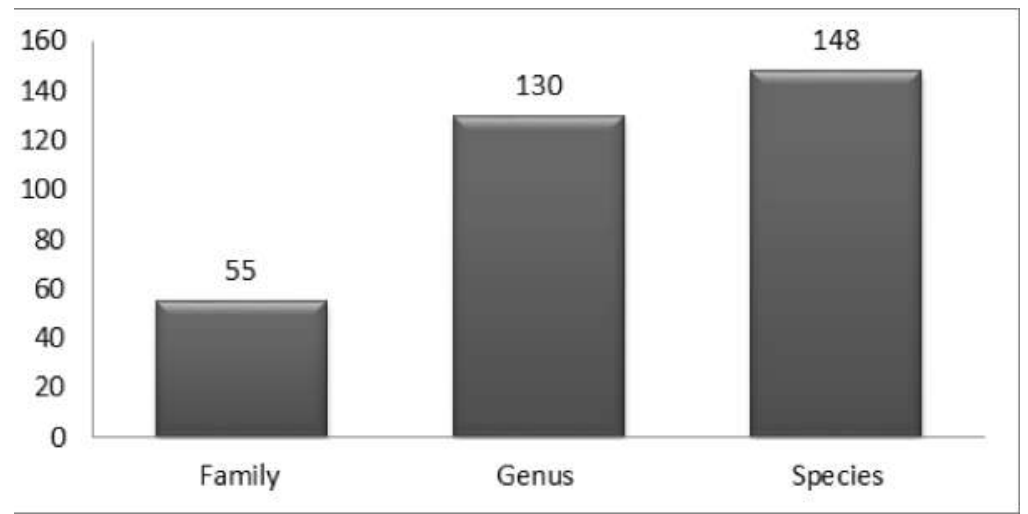

Figure 1. Showing total number of Family, Genus, and species in Reang Home Gardens

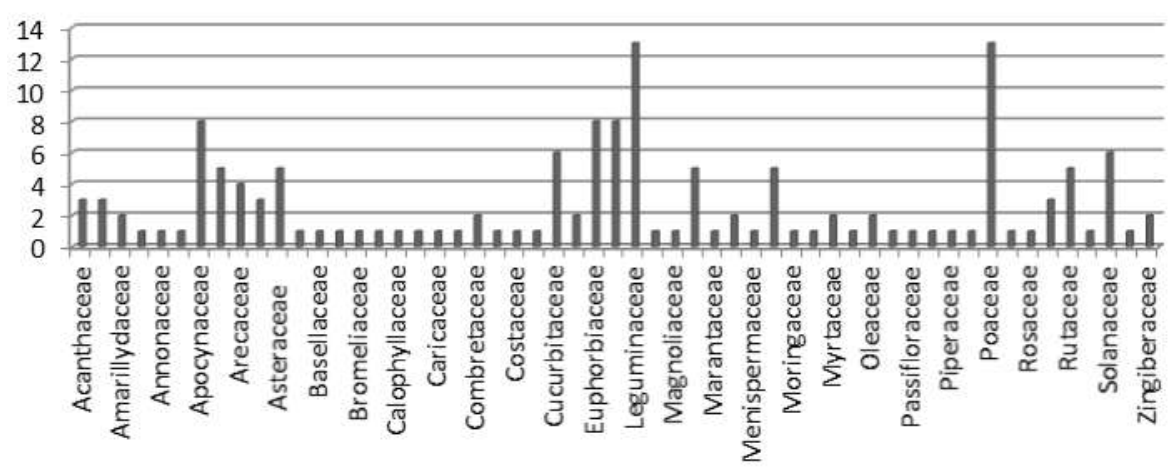

Figure 2. Representation of different plant families recorded from Reang Home Gardens

Out of 148 plant species herbs constitutes 69 species, trees 32 , small trees 15 , shrubs 16, under shrub 3, climbers 11 and 2 woody climbers were recorded [Figure 3]. 40\%of the Reang Home Garden plants are used as edible, $22 \%$ medicinal, $18 \%$ ornamental, $4 \%$ timber, $3 \%$ fire wood and the rest remain less than $3 \%$ which were construction material, broom making, ritual plants, masticator, stuffing material, fodder, rope making,multipurpose, cottage industry weed etc. [Figure 5]. Thus plants of the Reang Home garden show multiple uses and are maintained for meeting the requirements of their food, medicine, ornamental, construction materials, fuel wood etc.

Among the most frequently used food plants are Benincasa hispida, Cajanus cajan, Carica papaya, Dioscorea alata, Dioscorea bulbifera, Homalomena aromatica, Lagenaria siceraria, Musa paradisiaca, Phaseolus lunatus, Xanthosoma sagittifolium, Solanum melongena, Solanum torvum, Solanum violaceum, Citrus reticulata, Citrus hystrix, etc.

Reang people also sell Home garden products to supplement their income. Products found selling in the local market are shoots of Dendrocalamus longispathus, Bambusa tulda, Melocanna baccifer, young leaves of Lasia spinosa, Diplazium esculentum, Acacia pennata; root tubers of Manihot esculenta, Dioscorea glabra; petioles of Homalomena aromatica, Corm and daughter corm of Amorphophallus bulbifer, stem with rhizome of Alpinia nigra, leaves of Ocimum americanum, etc. are important (Table 2). 


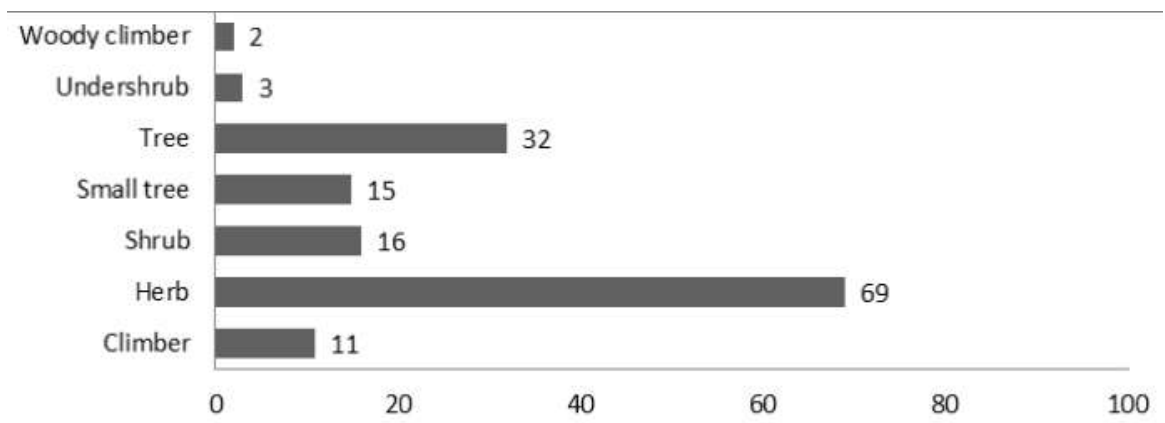

Figure 3. Plant species with various habit groups of Reang Home Gardens

Table 2. Result of market survey in of Kanchanpur area

\begin{tabular}{|c|c|c|c|}
\hline Plant Name & Family & Habit & Parts marketed \\
\hline Acacia pennata & Leguminosae & Liana & Leaves \\
\hline Alpinia allughas & Zingiberaceae & Herb & Stem, rhizome \\
\hline Amaranthus caudatus & Amaranthaceae & Herb & Whole plant \\
\hline Amorphophallus bulbifer & Araceae & Herb & Corm, petiole, young leaves \\
\hline Areca catechu & Arecaceae & Tree & Fruits \\
\hline Artocarpus heterophyllus & Moraceae & Tree & Fruits \\
\hline Bambusa tulda & Poaceae & Giant herb & Shoot \\
\hline Capsicum annuum & Solanaceae & Herb & Fruits \\
\hline Centella asiatica & Apiaceae & Herb & Leaves \\
\hline Citrus limon & Rutaceae & Shrub & Fruits \\
\hline Colocasia esculenta & Araceae & Herb & Root stock, stolon, petiole \\
\hline Corchorus olitorius & Tiliaceae & Herb & Leaves \\
\hline Cucumis sativus & Cucurbitaceae & Herb & Fruits \\
\hline Cucurbita pepo & Cucurbitaceae & Herb & Fruit, twigs \\
\hline Dendrocalamus longispathus & Poaceae & Giant herb & Shoot \\
\hline Dioscorea glabra & Dioscoreaceae & Herb & Root tuber \\
\hline Diplazium esculentum & Athyriaceae & Herb & Tender shoots \\
\hline Ensete glaucum & Musaceae & Herb & Young inflorescence \\
\hline Eryngium foetidum & Apiaceae & Herb & Leaves \\
\hline Hibiscus cannabinus & Malvaceae & Undershru & Leaves, young fruits \\
\hline Homalomena aromatica & Araceae & Herb & Petiole \\
\hline Lasia spinosa & Araceae & Herb & Young leaves \\
\hline Luffa acutangula & Cucurbitaceae & Herb & Fruits \\
\hline Manihot esculenta & Euphorbiaceae & Shrub & Root tuber, leaves \\
\hline Melocanna baccifera & Poaceae & Giant herb & Shoot \\
\hline Momordica charantia & Cucurbitaceae & Herb & Fruits, twigs \\
\hline Momordica dioica & Cucurbitaceae & Herb & Fruits \\
\hline Musa paradisiaca & Musaceae & Herb & Inflorescence, fruit \\
\hline Ocimum americanum & Lamiaceae & Herb & Leaves \\
\hline
\end{tabular}


Dipti Das \& B.K. Datta 217

\begin{tabular}{|l|l|l|l|}
\hline Plant Name & Family & Habit & Parts marketed \\
\hline Solanum melongena & Solanaceae & Undershrub & Fruits \\
\hline Solanum torvum & Solanaceae & Undershrub & Fruits \\
\hline $\begin{array}{l}\text { Trachyspermum } \\
\text { roxburghianum }\end{array}$ & Apiaceae & Herb & Leaves, \\
\hline Trichosanthescucumerina & Cucurbitaceae & Herb & Fruits \\
\hline Vigna unguiculata & Leguminosae & Herb & Fruits \\
\hline Zingiber officinale & Zingiberaceae & Herb & Rhizome, plant body \\
\hline
\end{tabular}

During this study $22 \%$ of the total plant species were found to be used as medicine. Most Reang peoples rely on folk health care traditions as they are residing in remote areas and for their socio-economic condition. They depend on traditional healers for treating various ailments using different plants, which is locally available and are inexpensive. Among the medicinal plants Aloe vera, Alpinia nigra, Alternanthera brasiliana, Asparagus racemosus, Ayapana triplinervis, Cheilocostus speciosus, Oroxylum indicum, Plumbago zeylanica, Rauvolfia serpentina, Solanum violaceum and Stephania glabra are important (Table 3). Domestication of wild plants in Home Gardens for medicine is an important activity in Xishuangbanna in China and other tropical regions (Gao et al. 2012). In the traditional Home gardens of North East India medicinal plants were second largest use category (Sahoo et al. 2010). Similar findings were observed in the Home Gardens of Assam after vegetable and fruit constituents. Medicinal plants were the most dominant species (Devi \& Das 2010). Medicinal plants in Home gardens were documented to be second important plant group in Sri Lanka and Bangladesh also (Mustafa et al. 2002).

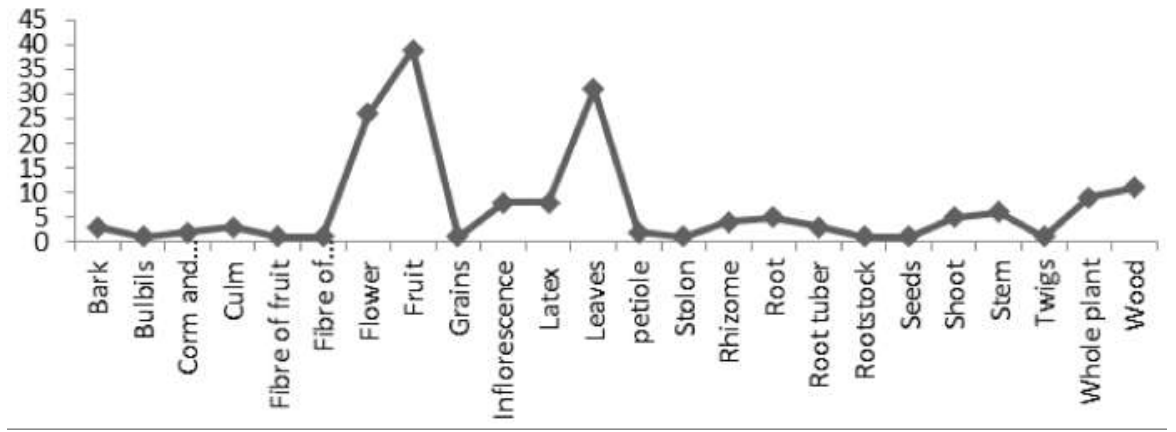

Figure 4. Different plant parts used by Reang tribe of North Tripura

Table 3. Ethnomedicinal uses of recorded plants

\begin{tabular}{|l|l|l|}
\hline \multicolumn{1}{|c|}{ Plant name } & Parts used & Mood of use \\
\hline Achyranthes aspera & Whole plant & Helps in increasing appetite and lactation \\
\hline Acmella paniculata & Inflorescence & Toothache \\
\hline Aloe vera & Leaves & $\begin{array}{l}\text { Used in a herbal mixture that cures menstrual } \\
\text { disorder }\end{array}$ \\
\hline Alpinia nigra & Stem & Cures liver disorder \\
\hline Alstonia scholaris & Bark & Used to cure fever. \\
\hline Alternanthera brasiliana & Leaves & $\begin{array}{l}\text { Mixed together with Plumbago and Datura } \\
\text { leaves and used for abortion }\end{array}$ \\
\hline Ananas comosus & Fruits, leaves & Used as anthelmintics \\
\hline Andrographis paniculata & Leaves & Juice helps to treat stomachache \\
\hline
\end{tabular}


218 Plant diversity in Reang Home Gardens in Tripura
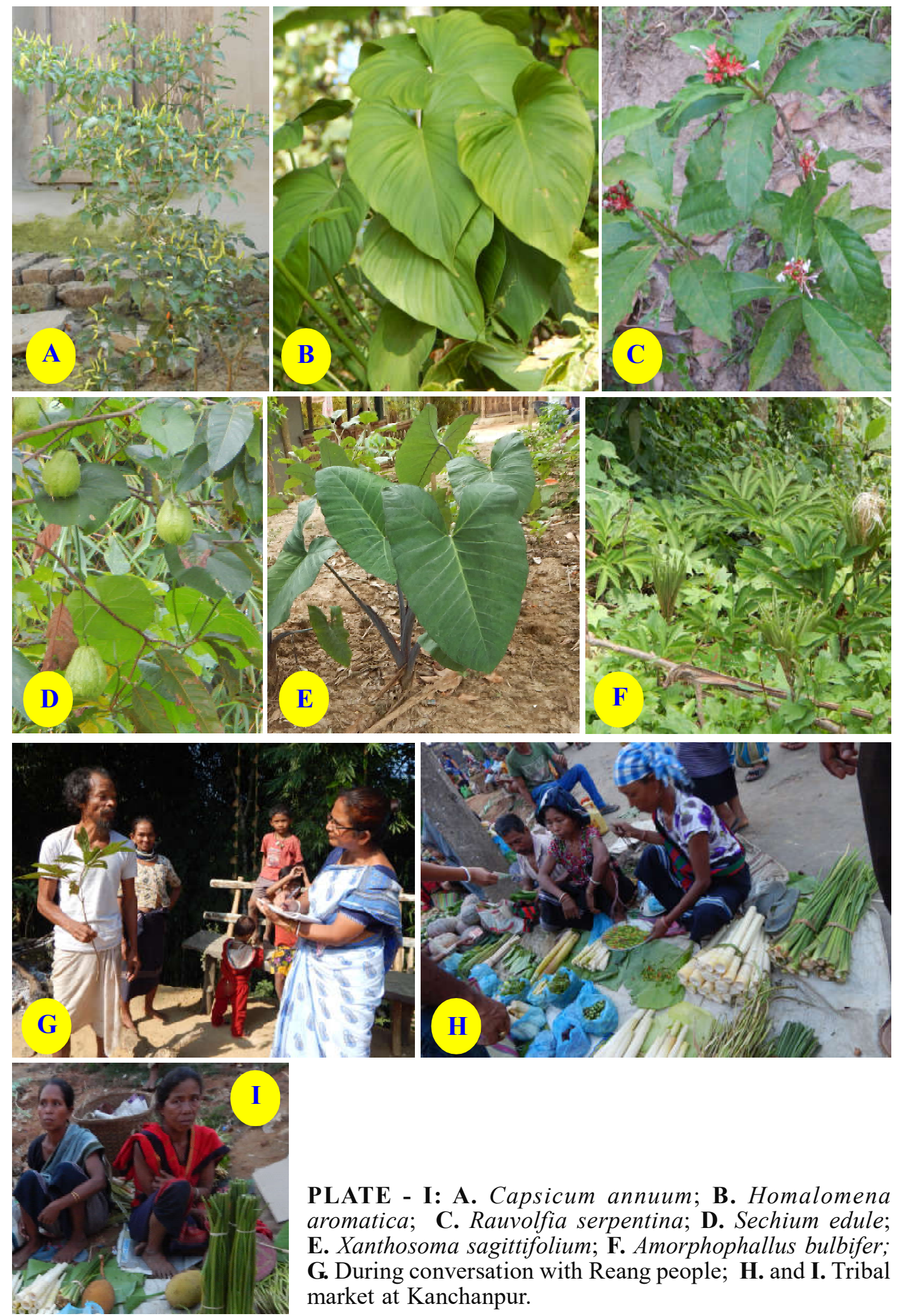

PLATE - I: A. Capsicum annuum; B. Homalomena aromatica; C. Rauvolfia serpentina; D. Sechium edule; E. Xanthosoma sagittifolium; F. Amorphophallus bulbifer; G. During conversation with Reang people; H. and I. Tribal market at Kanchanpur. 
Dipti Das \& B.K. Datta 219

\begin{tabular}{|c|c|c|}
\hline Plant name & Parts used & Mood of use \\
\hline Annona squamosa & Fruits, leaves & $\begin{array}{l}\text { Extract used to treat diarrhoea (fruits) and } \\
\text { dysentery (leaves) }\end{array}$ \\
\hline Asparagus racemosus & Root & Smashed and applied to in fever \\
\hline Ayapana triplinervis & Leaves & $\begin{array}{l}\text { Squashed and applied to cure eye infection; also } \\
\text { in cuts and wounds }\end{array}$ \\
\hline Bryophyllum pinnatum & Leaves & Helps to improve appetite \\
\hline Cheilocostus speciosus & Rhizome & Juice used to cure asthma and bronchitis \\
\hline Citrus hystrix & Fruit & Digestive stimulant \\
\hline Clerodendrum infortunatum & Root & Juice is used to treat blood dysentery \\
\hline Curcuma longa & Rhizome & Used to treat Jaundice \\
\hline Datura metel & Leaves & Used for abortion \\
\hline Euphorbia hirta & Young whole plant & Helps to increase lactation \\
\hline Euphorbia nerifolia & Latex, leaves & $\begin{array}{l}\text { Latex and leaf juice mixed with honey and used } \\
\text { to treat cough and bronchitis }\end{array}$ \\
\hline Garuga pinnata & Leaves & $\begin{array}{l}\text { Boiled leaf used in bathing as a treatment of } \\
\text { measles and pox }\end{array}$ \\
\hline Hemidesmus indicus & Root & Juice used to treat urinary infections \\
\hline Hyptis capitata & Leaves & Juice to treat fever \\
\hline Jatropha curcas & Latex & Applied in toothache \\
\hline Jatropha gossypiifolia & Latex & To treat cuts and wounds \\
\hline Justicia gendarussa & Leaves & Squashed and used in cuts and wounds \\
\hline Maranta arundinacea & Rhizome & Powdered and boiled, given in stomach disorder \\
\hline Melia azedarach & Leaves & Juice mixed with honey and used to treat eczema \\
\hline Morinda angustifolia & Root & $\begin{array}{l}\text { Paste used to treat smallpox and urinary } \\
\text { infection }\end{array}$ \\
\hline Morus australis Poir. & Fruits & Taken in fever \\
\hline Nyctanthes arbor-tristis & Flowers,leaves & As anthelmintic \\
\hline Ocimum tenuiflorum & Leaves & To treat common cold \\
\hline Oldenlandia verticillata & Leaves & $\begin{array}{l}\text { Juice used to treat woman in confinement and pox } \\
\text { patient }\end{array}$ \\
\hline Oroxylum indicum & Fruits, bark & Barks to treat jaundice \\
\hline Pandanus amaryllifolius & Leaves & In asthma \\
\hline Plumbago zeylanica & Leaves & In gynaecological disorder \\
\hline Rauvolfia serpentina & Roots & Smashed and taken in stomachache \\
\hline Sansevieria trifasciata & Rhizome & In gynaecological disorders \\
\hline Senna siamea & Root & To treating jaundice \\
\hline Solanum violaceum & Fruits & Smashed and consume during stomach ache \\
\hline Stephania glabra & Leaves & Smashed and used as ointment during headache \\
\hline Terminalia bellirica & Fruits & Used in hepatitis, bronchitis and asthma \\
\hline
\end{tabular}




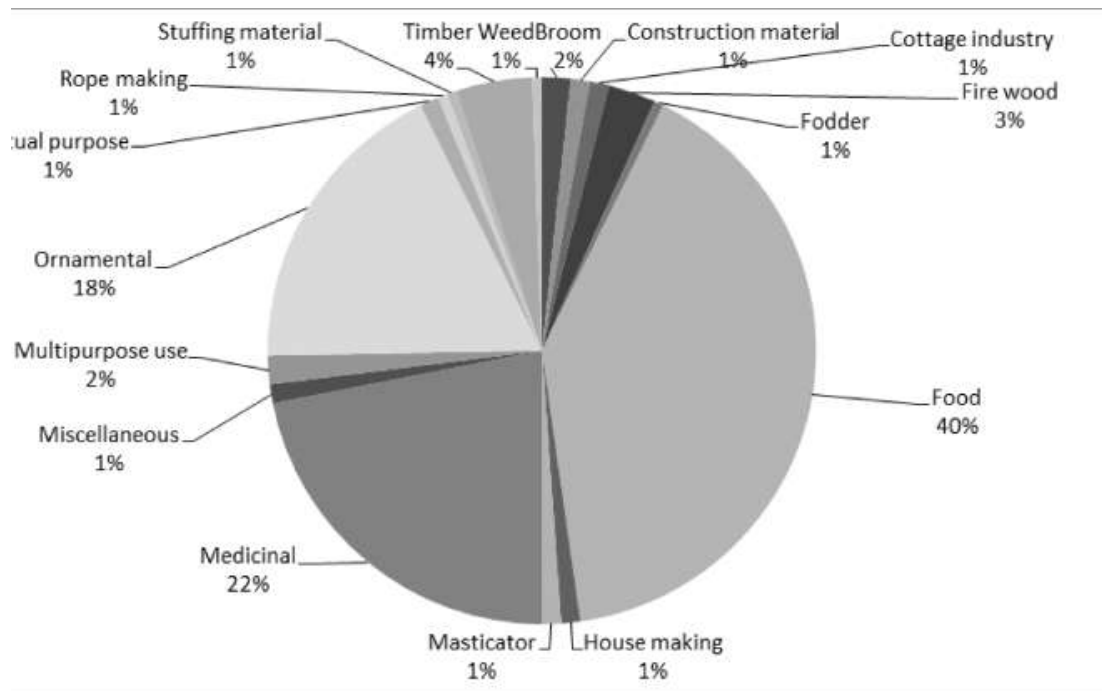

Figure 5. Plants with different conomic categories recorded from Reang Home Gardens

Local markets are the places where home garden products were also sold. During the local market survey at Kanchanpur and its adjoining areas, different plant parts belonging to 35 species were observed and recorded. Amongst them, large amount of young-shoots of different bamboo species, different types of aroids, cucurbits etc along with other plant products were generally found. Corm of Amorphophallus bulbifer, young shoot of Melocanna baccifera, daughter corm or cormel of Colocasia esculenta and petiole of Homalomena aromatica were the chief selling component.

\section{CONCLUSION}

The present initiative is the first attempt to conduct a study on the Home gardens of the Reang tribe of Tripura. From the above discussions, it was revealed that production system in Home Gardens of the study area was livelihood requirement and traditional knowledge, besides these they have additional income from Home Garden plant products.. They are practicing Home Gardens mainly to ensure food security and folk health care besides supplementing their income. This is also helping the Home garden owners in maintaining well being of their family.

\section{Acknowledgements}

Authors are grateful to Reang Home garden owners for allowing us to study in their Home gardens and also for sharing their valuable knowledge. Special thanks to Shri. Nantu Ranjan Das, the then SDM of Kanchanpur Subdivision for his help during the field survey. We are also grateful to Smt. Parbati Reang and Shri Raising Reang, health practitioner of Reang tribe for sharing their knowledge.

\section{LITERATURE CITED:}

Abdoellah, O. S. 1990. Homegardens in West Java and their future development. In: Landauer K. \& Brazil M. (eds.), Tropical Homegardens, United Nations University Press, Tokyo. Pp. $69-79$.

Acharyya, R.K. 1999. Insight into the REANGS. Tribal Research \& Cultural Institute, Govt. of Tripura. 
Agelet, A.; Angels, B.M. \& Valles, J. 2000. Home gardens and their role as a main source of medicinal plants in mountain regions of Catalonia (Iberian Peninsula). Econ. Bot. 54: $295-309$.

Bajpai, S.; Sharma, A.K. \& Kanungo,V.K. 2013. Traditional Home gardens: A preserve of medicinal plants. Intn. J. Herbal Med. 1(2): 152 - 161.

Borthakur, S.K.; Sarma, T.R.; Nath, K.K. \& Deka, P. 1998. The house gardens of Assam: a traditional Indian experience of management and conservation of biodiversity-I. Ethnobotany. 10: 32 - 37.

Das, T. \& Das, A.K. 2005. Inventorying plant diversity in Home garden - A case study of Barak valley, Assam, northeast India. Curr. Sci. 98: 155 - 163.

Deb, D.B. 1981-1983. The Flora of Tripura State. Vols. 1 \& 2. Today \& Tomorrow's Printers and Publishers, New Delhi.

Devi, N.L. \& Das, A.K. 2010. Plant species diversity in the traditional Home gardens of Meitei community : A case study from Barak Valley, Assam. J. Trop. Agri. 48: 40 - 43.

Devi, N.L. \& Das, A.K. 2013. Diversity and utilization of tree species in Meitei Home gardens of Barak Valley, Assam. J. Env. Biol. 34: 211 - 217.

Fernandes, E.C.M. \& Nair, P.K.R. 1986. An evaluation of the structure and function of tropical Home gardens. Agricultural Syst. 21: 279 - 310.

Gan Choudhury, J. 2011. The Reangs of Tripura. Tribal Research \& Cultural Institute, Govt. of Tripura.

Gao, J.; He, T. \& Li, Q.M. 2012. Traditional home-garden conserving genetic diversity: a case study of Acacia pennata in southwest China, Conserv. Genet. 13(4): $891-898$.

Gautam, R.; Suwal, R. \& Shrestha, P.K. 2004. Enhancing the contribution of home gardens to on-farm management of plant genetic resources and to improve the livelihoods of Nepalese farmers: Findings of baseline surveys of four project sites (Jhapa, Ilam, Rupandehi and Gulmi). Working Paper, Local Initiatives for Biodiversity, Research and Development (LIBIRD), Pokhara, Nepal. Pp. $51-65$.

Hooker, J. D. 1872-1897. The Flora of British India. Vol. 1-7. L. Reev and Co, Ashford, London.

Jain, S.K. \& Rao, R.R. 1977. A Handbook of Field and Herbarium Methods. Today and Tomorrow's Printers and Publishers, New Delhi.

Jose, D. \& Shanmugaratnam, N. 1993. Traditional Home gardens of Kerala: a sustainable human ecosystem. Agrofor. System. 24: 203 - 213.

Kabir, M.E. \& Webb, E.L. 2008. Floristics and structure of south western Bangladesh Home gardens. The Intn. J. Biodiv. Sci. Manag. 4 (1): $54-64$.

Kala, C.P. 2010. Home gardens and Management of Key Species in the Pachmarhi Biosphere Reserve of India. J. Biodiv. 1(2): $111-117$.

Kanjilal, U.N.; Kanjilal, P.C.; Das, A.; Dey, R.N. \& Bor, N.L. 1934-1949. Flora of Assam. Vol. I-V. Government press, Shilong.

Kumar, B.M.; George, S.J. \& Chinnamani, S. 1994. Diversity, structure and standing Stock of wood in the Home gardens of Kerala in peninsular India. Agrofor. System. 25: 243 - 262.

Michon, G. \& Mary, F. 1994. Conversion of traditional village gardens and new economic strategies of rural households in the area of Bogor, Indonesia. Agrofor. System. 25:31 -58 . 
Leiva, J.M.; Azurdia, C. \& Ovando, W.2001. Contributions of home gardens to in situ conservation in traditional farming systems - Guatemalan component. In: Watson, J.W. $\&$ Eyzaguirre, P.B. (eds) Proceedings of the second international home gardens workshop. Bioversity international, Rome, Italy, pp $56-72$.

Mohan, S.; Nair, P.K.R. \& Long, A.J. 2007. An assessment of ecological diversity in Home gardens: A case study from Kerala state, India. J. Sust. Agri. 29: 135 - 153.

Millat-E-Mustafa, M ; Teklehaimanot, Z.\& K.o. Haruni, A. 2002. Traditional uses of perennial homestead garden plants in Bangladesh. Forests, Trees and Livelihoods. 12: 235 - 256.

Nair, M.A. \& Sreedharan, C. 1986. Agro forestry farming systems the homesteads of Kerala, southern India. Agrofor. System. 4: 339 - 363.

Pandey, C.B.; Rai, R.B.; Singh, L. \& Singh, A.K. 2007. Home gardens of Andaman and Nicobar, India. Agrofor. System. 92: 1 - 22.

Perera, A.H. \& Rajapakse, R.M.N. 1991. A baseline study of Kandyan forest gardens of Sri Lanka: Structure composition and utilization. Forest Ecology and Management. 45: $269-280$.

Peyre A.; Guidal A.; Wiersum K.F. \& Bongers F. 2006. Dynamics of homegarden structure and function in Kerala, India. Agrofor. System. 66: $101-115$.

Prain, D. 1903. Bengal Plants. Vol. I \& II. Repd. ed. BSI, Calcutta.

Sahoo, U.K.; Rocky, P.; Vanlalhriatpuia, K. \& Upadhyaya, K. 2010. Structural diversity and functional dynamism of traditional Home gardens of north-east India. The Bioscan (Special issue). 1: $159-171$.

Samati, H. 2004. Kitchen garden plants of Pnar tribe of Jantia hill district. Ethnobotany. 16 (1\& 2): $125-130$.

Soemarwoto, O. 1987. Homegardens: A traditional agro forestry system with a promising future. In: Steppler H.A. \& Nair P.K.R. (eds.), Agro forestry: A Decade of Development, ICRAF, Nairobi. Pp. 157 - 170.

Talemos, S.; Sebsebe, D. \& Zemede, A. 2014. Home gardens of Wolayta, Southern Ethiopia: An ethno botanical profile. J. Biol. Series. 1(1): 9-25.

Tangjang, S. \& Arunachalam, A .2009. Role of traditional Home garden systems in north India. Indian J. Trad. Knowl. 8(1): 47 - 50.

www.theplantlist.org, Version 1.1. 2013.

Thomas, A. \& Kurien, S. 2013. Homegardens of Kerala: Structural Configuration and Biodiversity. Paripex - Indian J. Res. 2(1): 133 - 135.

Watson, J.W. \& Eyzaguirre, P.B. 2002. Proceedings of the Second International Home gardens Workshop: Contribution of Home gardens to in situ conservation of plant genetic resources in farming systems, 17-19 July2001, Witzenhausen, Federal Republic of Germany. International Plant Genetic Resources Institute, Rome, Pp. 4 - 5. 